\title{
Prevalência de fragilidade entre idosos baianos assistidos na Atenção Básica
}

\author{
Prevalence of frailty among elderly bahians assisted in Primary Care \\ Prevalencia de la fragilidad entre ancianos bahianos asistidos en Atención Primaria
}

Recebido: 10/01/2022 | Revisado: 13/01/2022 | Aceito: 20/01/2022 | Publicado: 22/01/2022

\author{
Joana Trengrouse Laignier de Souza \\ ORCID: https://orcid.org/0000-0001-5793-6043 \\ Universidade Federal da Bahia, Brasil \\ E-mail: joana.trengrouse@ufba.br \\ Cirléia Alves Silva \\ ORCID: https://orcid.org/0000-0002-1000-6348 \\ Universidade Federal da Bahia, Brasil \\ E-mail: cid-alves@hotmail.com \\ Dóris Firmino Rabelo \\ ORCID: https://orcid.org/0000-0002-0791-7781 \\ Universidade Federal do Recôncavo da Bahia, Brasil \\ E-mail: drisrabelo@ufrb.edu.br
}

\begin{abstract}
Resumo
A síndrome da fragilidade é uma condição multifatorial que resulta em vulnerabilidade clínica e mortalidade. Objetivo: Verificar a prevalência de fragilidade e fatores associados entre pessoas idosas usuárias da Unidade Básica de Saúde (UBS). Método: Estudo observacional de corte transversal, com 354 idosos (as) de uma UBS da Bahia. Os dados foram obtidos pelos prontuários contendo o Índice de Vulnerabilidade Clínico-Funcional e dados sociodemográficos, para análise utilizaram-se ferramentas estatísticas com análises descritivas, bivariada e múltipla. Resultados: Os idosos avaliados tinham idade média de 72,4 anos (DP 8,35), em sua maioria autodeclarados negros, mulheres, com baixa escolaridade e residindo apenas com o cônjuge. Houve prevalência de fragilidade em 15,8\% dos idosos, pré-fragilidade em $32,2 \%$ e robustez em $52 \%$. Constatou-se associação estatisticamente relevante entre fragilidade e dependência para atividades diárias e comorbidades múltiplas. A fragilidade foi mais frequente entre as mulheres, os mais velhos, os com baixa escolaridade, os com coabitação com outros familiares ou só com cônjuge e os com autopercepção de saúde negativa. Conclusão: Reconhecer os fatores associados é indispensável para elaboração de políticas efetivas para pessoas idosas.
\end{abstract}

Palavras-chave: Idoso; Fragilidade; Serviços de saúde para idosos; Fluxo de trabalho; Atenção Primária à Saúde.

\begin{abstract}
Frailty syndrome is a multifactorial condition that results in clinical vulnerability and mortality. Objective: To verify the prevalence of frailty and associated factors among elderly users of a Basic Health Unit (UBS - Unidade básica de saúde). Method: Cross-sectional observational study with 354 elderly people from a UBS in Bahia. Data were obtained from medical records containing the Clinical-Functional Vulnerability Index and sociodemographic data. Statistical tools were used to analyse with descriptive, bivariate and multiple methods. Results: The elderly evaluated had a mean age of 72.4 years (SD 8.35), mostly self-declared black, women, with low level of education and living only with their spouse. There was a prevalence of frailty in $15.8 \%$ of the elderly, pre-frailty in $32.2 \%$ and robustness in $52 \%$. A statistically relevant association was found between frailty and dependence for daily activities and multiple comorbidities. Frailty was more frequent among women, the elderly, those with low level of education, those living with other family members or just a spouse, and those with a negative self-perception of health. Conclusion: Recognizing the associated factors is essential for the development of effective policies for older people.
\end{abstract}

Keywords: Aged. Frailty; Health services for the aged; Workflow; Primary Health Care.

\section{Resumen}

El síndrome de fragilidad es una condición multifactorial que resulta en vulnerabilidad clínica y mortalidad. Objetivo: Verificar la prevalencia de fragilidad y factores asociados entre los usuarios mayores de la Unidad Básica de Salud (UBS). Método: Estudio observacional transversal con 354 ancianos de una UBS en Bahía. Los datos se obtuvieron de historias clínicas que contenían el Î́ndice de Vulnerabilidad Clínico-Funcional y datos sociodemográficos, se utilizaron herramientas estadísticas para el análisis con análisis descriptivo, bivariado y múltiple. Resultados: Los ancianos evaluados tenían una edad media de 72,4 años (DE 8,35), en su mayoría mujeres autodeclaradas negras, con baja escolaridad y viviendo solo con su cónyuge. Hubo una prevalencia de fragilidad en el 15,8\% de los ancianos, prefragilidad en el $32,2 \%$ y robustez en el $52 \%$. Se encontró asociación estadísticamente relevante entre fragilidad y dependencia para las actividades diarias y múltiples comorbilidades. La fragilidad fue más frecuente entre las mujeres, 
las personas mayores, las personas con bajo nivel educativo, las que vivían con otros miembros de la familia o simplemente un cónyuge y las que tenían una autopercepción negativa de la salud. Conclusión: Reconocer los factores asociados es fundamental para el desarrollo de políticas efectivas para las personas mayores.

Palabras clave: Anciano; Fragilidad; Servicios de salud para personas mayores; Flujo de trabajo; Primeros auxilios.

\section{Introdução}

Considerando o conceito ampliado de saúde e a definição trazida pela Política Nacional de Saúde da Pessoa Idosa (PNSPI), a saúde dos(as) idosos(as) deve ser avaliada com base no funcionamento de sistemas funcionais que interagem entre si a fim de garantir a manutenção da capacidade individual de decisão e comando sobre suas ações (autonomia) e a capacidade de realização por meios próprios (independência). Tais sistemas funcionais descritos são: cognição, humor, mobilidade e comunicação (Brasil, 2021; Moraes, 2012). Com a pandemia da COVID-19 em 2020, evidenciou-se uma piora nas condiç̃̃es de saúde de pessoas idosas na ordem de 21,9\% (Romero et al, 2021), o que torna ainda mais urgente a intensificação de políticas públicas de proteção à essa população.

A Síndrome da Fragilidade (SF) é reconhecida como um problema de saúde pública, visto suas consequências clínicas e sociais. É uma condição inicialmente subclínica, multifatorial, associada ao aumento da vulnerabilidade a estressores internos e externos de naturezas diversas. A identificação e o encaminhamento adequados constituem uma potente estratégia para os sistemas de saúde evitarem desfechos desfavoráveis, visto que pode impactar na prevenção de perda funcional e cognitiva, na manutenção do autocuidado, redução do risco de quedas, institucionalização e hospitalizações (Castro-Costa et al., 2018; Faller et al., 2019; Placideli et al., 2020). Pessoas idosas são usuárias frequentes do sistema público de saúde e assistência social, sistemas que são desenhados para a assistência órgão-específica ou doença-específica e, portanto, não estão preparados para o cuidado de condições clínicas complexas e multidimensionais de idosos(as) frágeis (Kojima, 2019).

Tanto no Brasil quanto em diversos países observa-se uma grande variabilidade nos estudos de prevalência de fragilidade entre pessoas idosas, o que inclui os modelos de avaliação e os instrumentos de triagem (Maia et al. 2020). Independentemente dos instrumentos utilizados para medir fragilidade, pessoas idosas frágeis tendem a apresentar pior qualidade de vida, uma vez que a SF frequentemente está associada a perda de peso, fadiga, fraqueza global, alterações de marcha, imobilidade, humor deprimido e déficit cognitivo (Kojima et al, 2016). No contexto da atenção primária, estudos mostraram que a SF está associada a condições sociodemográficas, polipatologia, polifarmácia, pior saúde mental, comprometimento cognitivo, pior capacidade funcional, quedas, internação, incontinência urinária, autopercepção negativa da saúde (Freitas et al, 2020; Maia et al. 2020; Placideli et al., 2020; Oliveira et al, 2021).

Esse cenário mostra a necessidade de ampliar os estudos sobre o tema em contextos diferentes, por exemplo, os que incluam pessoas idosas do território baiano e a variável raça. Envelhecer na Bahia pode ser desafiador, dadas as condições sociodemográficas e de saúde resultantes de uma cultura escravocrata e das desigualdades econômicas e raciais em nosso país. Cerca de $71 \%$ das pessoas idosas baianas se autodeclaram negras, um número expressivamente maior que a proporção total de idosos brasileiros que somam 42\% (IBGE, 2010). Contudo, raramente a raça/cor da pele é considerada nos estudos embora ela esteja associada a piores desfechos em saúde de pessoas idosas (Silva, 2019; Silva et al., 2018).

A SF pode ser prevenida e é passível de reabilitação, o que coloca a Atenção Básica (AB) em posição estratégica para a utilização de instrumentos para o seu reconhecimento (Faller et al., 2019). Estratificar os idosos da comunidade segundo sua vulnerabilidade clínico-funcional pode ser um caminho para incluir a Saúde da Pessoa Idosa de maneira mais eficaz entre as ações programáticas da APS. Seria útil para fins de avaliação de risco, facilitando a elaboração de planos de cuidados individualizados e ações programáticas coletivas e realizando a gestão da demanda programada a fim de priorizar os(as) idosos(as) que necessitam de atendimento diferenciado. Além de promover a melhora da qualidade do atendimento clínico do(a) idoso(a), uma vez que permite a identificação de alterações em múltiplos domínios, direcionando o profissional na investigação 
diagnóstica e no estabelecimento de medidas de promoção, prevenção, reabilitação e de cuidados paliativos (Brasil, 2019).

Assim, considera-se fundamental instituir, no âmbito da Atenção Básica, um programa de rastreamento de fragilidade na população idosa, a fim de reconhecer o perfil dos(as) idosos(as) no território, criar um fluxograma de atendimento interno e externo, promover o diálogo com as diferentes instâncias governamentais e a comunidade científica. Nesse sentido, o presente estudo tem por objetivo identificar a prevalência de fragilidade e fatores associados entre os idosos do território de Unidade Básica de Saúde (UBS) de Vitória da Conquista - Bahia, bem como as alterações mais frequentes nas dimensões de saúde.

\section{Metodologia}

\section{Desenho do estudo}

Trata-se de estudo observacional de corte transversal, de abordagem quantitativa com base em dados secundários obtidos dos prontuários de usuários (as) idosos (as) de uma unidade de Estratégia de saúde da família do município de Vitória da Conquista - BA.(Gil, 2002; Lakatos \& Marconi, 2003; Fontelles et al., 2009)

\section{Contexto do estudo}

Vitória da Conquista é o terceiro maior município do estado da Bahia e o quarto do interior do Nordeste. Segundo os últimos dados censitários, a população idosa de Vitória da Conquista somava 26.059 habitantes e representava quase 10\% da população à época (IBGE, 2011). Segundo dados do SISAP-idoso, $46 \%$ dos(as) idosos(as) em Vitória da Conquista são analfabetos, cerca de $62 \%$ apresentam alguma deficiência, pouco mais de $10 \%$ moram sozinhos e $70 \%$ possuem renda nominal de até um salário mínimo. Considerando a velocidade de crescimento do município nos últimos 9 anos e a sua importância na macrorregião sudoeste, o desenvolvimento de políticas de saúde pública voltadas para a população idosa constitui tema de altíssima relevância. Cerca de 66\% das pessoas idosas no município encontram-se cadastrados na Estratégia de Saúde da Família (ESF) (FIOCRUZ, 2011).

Foi selecionada para o estudo uma Unidade de Estratégia de Saúde da Família do município, que fica localizada em um bairro periférico da cidade, e que conta com uma equipe do Programa de Educação pelo Trabalho para a Saúde (PET-Saúde) atuando com o projeto "Educação interprofissional e práticas colaborativas na produção do cuidado integral ao idoso na APS". Segundo dados do eSUS disponibilizados pela unidade, o número total de usuários cadastrados em 2019 era de 5876 cidadãos ativos, sendo um total de 1105 usuários com 60 anos ou mais, o que representa 18,8\% da população descrita.

\section{Amostra}

Foram incluídos indivíduos acima de 60 anos de idade da área adstrita à unidade cujas fichas do prontuário encontravamse corretamente preenchidas em todos os campos de avaliação da fragilidade. Foram excluídos os prontuários de pessoas com idade inferior a 60 anos, aqueles cujas fichas não possuíam todos os campos de avaliação da fragilidade preenchidos ou preenchidos de forma incorreta. Partindo-se do número total de idosos (as) cadastrados na UBS em 2019 (1105), foi realizado cálculo do tamanho da amostra, considerando-se um nível de confiança de 99\% e erro amostral de 5\%, que resultou em 330 idosos com acréscimo de 7\%, antecipando possíveis perdas, totalizando 354 idosos (as).

\section{Variáveis e medidas}

Foram analisados os dados contidos no Questionário sociodemográfico (sexo, raça/cor, escolaridade e condição de moradia) e os dados do Índice de Vulnerabilidade Clínico-Funcional (IVCF-20) para avaliação de fragilidade e estratificação dos idosos. O IVCF é constituído por 20 questões distribuídas em 8 seções que buscam contemplar as principais dimensões de saúde da pessoa idosa. Desta forma, foram utilizadas como variáveis de estudo cada uma dessas seções e suas respectivas 
subdivisões: 1. Faixa etária; 2. Autopercepção da saúde; 3. Atividades de Vida Diária: a) Instrumentais e b) Básicas; 4. Cognição; 5. Humor; 6. Mobilidade: a) Alcance, preensão e pinça, b) Capacidade Aeróbica e/ou muscular, c) Marcha e d) Continência Esfincteriana; 7. Comunicação: a) Visão e b) Audição, 8. Comorbidades Múltiplas. Além das variáveis acima listadas, serão consideradas as pontuações finais: sem fragilidade, pré-frágeis e frágeis.

\section{Coleta de dados e aspectos éticos}

Os dados que compuseram o presente estudo foram obtidos a partir dos prontuários de usuários(as) idosos(as) de uma unidade de Estratégia de saúde da família do município de Vitória da Conquista - BA, acessados pelo banco de dados do Programa Municipal de Atenção à Saúde do Idoso no Pólo de Educação da Atenção Básica de Vitória da Conquista.

Respeitando o estabelecido na Resolução do Conselho Nacional de Saúde 466/12, entende-se que o presente estudo prescinde de Termo de Consentimento Livre e Esclarecido (TCLE) por se tratar de coleta de dados secundários, oriundos do processo assistencial da equipe de saúde da família que compõem o prontuário do cidadão, não havendo contato direto entre o pesquisador e os idosos participantes. O presente estudo ocorreu entre os meses de maio e dezembro de 2020, somente após aprovação do Comitê de Ética em Pesquisa da Escola de Enfermagem da UFBA parecer nº 3.969.730.

\section{Procedimentos de análise de dados}

A digitação e tabulação dos dados coletados, bem como a análise foi realizada através da utilização do software STATA® da StataCorp LLC, versão 14.2. Para a análise de dados foi utilizada a análise estatística descritiva básica univariada (médias, medianas, distribuição de frequência, percentuais, correlações, medidas de dispersão e medidas de tendência central), e multivariada para a correlação de múltiplas variáveis simultaneamente. Foram utilizados o teste estatístico Qui-quadrado para variáveis qualitativas nominais e de Mann-Whitney para as qualitativas ordinais. Utilizamos o modelo de regressão logística simples e com ajuste para as medidas de associação.

\section{Resultados}

Os(as) idosos(as) tinham idade média de 72,4 anos (DP 8,35), sendo que a maioria era mulher, autodeclarada negra e com baixa escolaridade. As condições de moradia foram bem variáveis, e a maior parte se concentrou nos arranjos só com o cônjuge, só com filhos e com o cônjuge e filhos (Tabela 1). Quanto à fragilidade observou-se uma prevalência de fragilidade em $15,8 \%$ dos (as) idosos (as), pré-fragilidade em 32,2\% e 52\% foram considerados robustos. Para fins de análise, os (as) idosos (as) foram separados em dois grupos: robustos (sem fragilidade) e idosos(as) com algum grau de fragilidade (somatórios dos (as) idosos (as) frágeis e pré-frágeis). 
Tabela 1 - Medidas de tendência central, distribuição de características em idosos (as) da Unidade Básica de Saúde, Bahia, Brasil, $2020(\mathrm{n}=354)$.

\begin{tabular}{|c|c|}
\hline \multicolumn{2}{|c|}{ Idade } \\
\hline Média (DP) & $72,4(8,35)$ anos \\
\hline Mediana & 71 anos \\
\hline Mínima & 60 anos \\
\hline $25 \%$ & 65 anos \\
\hline $75 \%$ & 78 anos \\
\hline Máxima & 97 anos \\
\hline \multicolumn{2}{|c|}{ Sexo } \\
\hline Masculino & $135(38,1 \%)$ \\
\hline Feminino & $219(61,9 \%)$ \\
\hline \multicolumn{2}{|c|}{ Raça/Cor } \\
\hline Branco & $115(32,5 \%)$ \\
\hline Preta & $26(7,3 \%)$ \\
\hline Parda & $198(55,9 \%)$ \\
\hline Amarela & $3(0,9 \%)$ \\
\hline Indígena & $2(0,6 \%)$ \\
\hline Ignorado & $10(2,8 \%)$ \\
\hline \multicolumn{2}{|c|}{ Escolaridade } \\
\hline Não estudou & $76(21,5 \%)$ \\
\hline Estou até 4 anos & $120(33,9 \%)$ \\
\hline Entre 4 e 8 anos & $76(21,5 \%)$ \\
\hline 8 anos ou mais & $46(13 \%)$ \\
\hline Ignorado & $36(10,1 \%)$ \\
\hline \multicolumn{2}{|c|}{ Arranjo de Moradia } \\
\hline Sozinho & $33(9,3 \%)$ \\
\hline Só com cônjuge & $98(27,7 \%)$ \\
\hline Só com filhos & $59(16,7 \%)$ \\
\hline Com cônjuge e filhos & $58(16,4 \%)$ \\
\hline Só com netos & $10(2,8 \%)$ \\
\hline Com cônjuge e netos & $8(2,3 \%)$ \\
\hline Com cônjuge, filhos e netos & $13(3,7 \%)$ \\
\hline Com outros familiares & $12(3,4 \%)$ \\
\hline Com filhos e/ou netos e outros familiares & $30(8,5 \%)$ \\
\hline Outros arranjos de moradia & $7(1,9 \%)$ \\
\hline Ignorado & $26(7,3 \%)$ \\
\hline
\end{tabular}

Fonte: Elaborado pelos autores (2021).

A prevalência de fragilidade foi maior entre as mulheres, entre as pessoas idosas com baixa escolaridade (4 anos ou menos) e entre os(as) que coabitavam com outros familiares que não sozinhos ou só com cônjuge. Quanto à raça, em nossa amostra, não houve associação estatística para fragilidade (Tabela 2). 
Tabela 2 - Distribuição de características de idade, sexo, raça/cor, escolaridade e arranjo de moradia e suas medidas de associação com Fragilidade em idosos da UBS, Bahia, Brasil, 2020 (n=354).

\begin{tabular}{|c|c|c|c|c|}
\hline \multirow{2}{*}{$\begin{array}{l}\text { Características } \\
\text { Idade }\end{array}$} & \multicolumn{2}{|c|}{ Fragilidade } & \multirow[t]{2}{*}{ RP (IC 95\%) } & \multirow[t]{2}{*}{ P-valor } \\
\hline & $\operatorname{Sim} n(\%)$ & Não n(\%) & & \\
\hline 60-79 anos & $112(40,4 \%)$ & $165(9,6 \%)$ & 1,00 & $<0,01$ \\
\hline 80+ anos & $56(72,7 \%)$ & $21(27,3 \%)$ & $1,80(1,47 ; 2,19)$ & \\
\hline \multicolumn{5}{|l|}{ Sexo } \\
\hline Masculino & $53(39,3 \%)$ & $82(60,7 \%)$ & 1,00 & $<0,01$ \\
\hline Feminino & $115(52,5 \%)$ & $104(47,5 \%)$ & $1,34(1,05 ; 1,71)$ & \\
\hline \multicolumn{5}{|l|}{ Raça/cor } \\
\hline Brancos & $59(51,3 \%)$ & $56(48,7 \%)$ & 1,00 & $>0,01$ \\
\hline Não-Brancos & $109(45,6 \%)$ & $130(54,4 \%)$ & $0,89(0,71 ; 1,11)$ & \\
\hline \multicolumn{5}{|l|}{ Escolaridade } \\
\hline Mais de 4 anos & $48(39,3 \%)$ & $74(60,7 \%)$ & 1,00 & 0,01 \\
\hline 4 anos ou menos & $104(53,1 \%)$ & $92(46,9 \%)$ & $1,34(1,04 ; 1,74)$ & \\
\hline \multicolumn{5}{|l|}{ Arranjo de Moradia } \\
\hline Sozinho ou só com cônjuge & $48(36,6 \%)$ & $83(63,4 \%)$ & 1,00 & $<0,01$ \\
\hline Com outros familiares & $109(55,3 \%)$ & $88(44,7 \%)$ & $1,51(1,16 ; 1,95)$ & \\
\hline
\end{tabular}

RP: Razão de Prevalência; IC: Intervalo de Confiança; Para Moradia houve 26 ignorados. Fonte: Elaborado pelos autores (2021).

Observamos que a mediana das pontuações se eleva de 5 entre os (as) idosos (as) de 60 a 79 anos para 11 entre os(as) acima de 80 anos, sendo a associação entre idade acima de 80 anos e fragilidade estatisticamente significativa. Para obtenção da medida de associação ajustada entre fragilidade e a idade, foram selecionadas as covariáveis Sexo, Raça/cor e Polifarmácia (Tabela 5) como confundidoras, incluídas no modelo final de análise obtivemos RP 1,85 (1,52-2,10), demonstrando associação entre idade de 80 anos ou mais e fragilidade, com significância estatística $(\mathrm{p}<0,001)$.

Com relação aos domínios avaliados pelo instrumento IVCF-20, foram analisadas as suas correlações com a fragilidade de acordo com a faixa etária e o sexo, (Tabelas 3 e 4 respectivamente). Dentre os domínios que se correlacionaram estatisticamente com a fragilidade de acordo com as faixas etárias, observamos que houve associação entre a dependência para atividades de vida diária tanto básicas quanto instrumentais em idosos (as) acima de 80 anos, a mobilidade associada tanto a membros superiores quanto membros inferiores (alcance, preensão e pinça; capacidade aeróbica e muscular; e marcha), porém não houve associação quanto à continência. Houve associação estatisticamente significativa entre a comunicação, tanto visão quanto audição; bem como a presença de comorbidades múltiplas.

No que concerne às comorbidades múltiplas, observamos uma prevalência de 4,8\% de polipatologia, 25,1\% de polifarmácia e 4,8\% de internação recente na amostra total de indivíduos, demonstrando que a polifarmácia foi responsável por $83 \%$ das pontuações na dimensão de comorbidades múltiplas. Houve uma associação entre fragilidade e polifarmácia estatisticamente significativa, com risco relativo de 2,58 (IC 2,30;2,75; $\mathrm{p}<0,0001$ ).

Na análise multivariada (Tabela 5), por sua vez, quando ajustada para idade, sexo e raça/cor, o RR se eleva para 2,59 com IC 2,29;2,77 (p<0,0001). Houve associação entre polifarmácia e o sexo feminino (RR 1,77 IC 1,16;2,70 p<0,01). Já a presença de polipatologia associou-se à fragilidade com um risco relativo de 2,23 (IC95\% 1,98;2,51, p=0,00001) e o histórico de internação recente apresentou RR 1,94 (IC95\% 1,58;2,40, p=0,0006). 
Tabela 3 - Associação entre faixa etária os domínios do IVCF-20 em idosos (as)da UBS, Bahia, Brasil, 2020 (n=354).

\begin{tabular}{|c|c|c|c|}
\hline Faixa etária & $\begin{array}{c}\text { 60-79 anos } \\
\text { n }(\%)\end{array}$ & $\begin{array}{c}\text { 80+ anos } \\
\text { n }(\%)\end{array}$ & P-valor \\
\hline Autopercepção da saúde & & & 0,699 \\
\hline Excelente/Muito Boa/Boa & $137(49,5 \%)$ & $40(51,9 \%)$ & \\
\hline Regular/ruim & $140(50,5 \%)$ & $37(48,1 \%)$ & \\
\hline AIVD & & & 0,0001 \\
\hline Independente & $230(83 \%)$ & $38(49,4 \%)$ & \\
\hline Com dependência & $47(17 \%)$ & $39(50,6 \%)$ & \\
\hline ABVD & & & 0,0001 \\
\hline Independente & $270(97,5 \%)$ & $64(83,1 \%)$ & \\
\hline Com Dependência & $7(2,5 \%)$ & $13(16,9 \%)$ & \\
\hline Cognição & & & 0,788 \\
\hline Sem déficit & $168(60,6 \%)$ & $48(62,3 \%)$ & \\
\hline Com déficit & $109(39,4 \%)$ & $29(37,7 \%)$ & \\
\hline Humor & & & 0,24 \\
\hline Normal & $182(65,7 \%)$ & $45(58,4 \%)$ & \\
\hline Alterado & $95(34,3 \%)$ & $32(41,6 \%)$ & \\
\hline Alcance, Preensão e Pinça & & & 0,0004 \\
\hline Capaz & $257(92,8 \%)$ & $63(81,8 \%)$ & \\
\hline Incapaz & $20(7,2 \%)$ & $14(18,2 \%)$ & \\
\hline Capacidade Aeróbica e Muscular & & & 0,0001 \\
\hline Normal & $189(68,2 \%)$ & $30(39 \%)$ & \\
\hline Alterado & $88(31,8 \%)$ & $47(61 \%)$ & \\
\hline Marcha & & & 0,001 \\
\hline Normal & $191(68,9 \%)$ & $37(48 \%)$ & \\
\hline Alterada & $86(31,1 \%)$ & $40(52 \%)$ & \\
\hline Continência & & & 0,073 \\
\hline Normal & $224(80,9 \%)$ & $55(71,4 \%)$ & \\
\hline Alterada & $53(19,1 \%)$ & $22(28,6 \%)$ & \\
\hline Visão & & & 0,018 \\
\hline Sem problema incapacitante & $245(88,5 \%)$ & $60(86,2 \%)$ & \\
\hline Com problema incapacitante & $32(11,5 \%)$ & $49(13,8 \%)$ & \\
\hline Audição & & & 0,0001 \\
\hline Sem problema incapacitante & $256(92,4 \%)$ & $59(76,6 \%)$ & \\
\hline Com problema incapacitante & $21(7,6 \%)$ & $18(23,4 \%)$ & \\
\hline Comorbidades Múltiplas & & & 0,012 \\
\hline Não & $203(73,3 \%)$ & $45(58,4 \%)$ & \\
\hline Sim & $74(26,7 \%)$ & $32(41,6 \%)$ & \\
\hline Classificação Final & & & 0,0001 \\
\hline Robustos(as) & $164(59,2 \%)$ & $20(26 \%)$ & \\
\hline Pré-frágeis & $87(31,4 \%)$ & $27(35,1 \%)$ & \\
\hline Frágeis & $26(9,4 \%)$ & $30(38,9 \%)$ & \\
\hline
\end{tabular}

Fonte: Elaborado pelos autores (2021).

Quando analisados os domínios conforme o sexo, observa-se uma associação significativa entre a autopercepção de saúde e a presença de comorbidades múltiplas e a fragilidade. Quanto a autopercepção de saúde, podemos observar que houve associação estatisticamente significativa entre o sexo feminino e autopercepção negativa da saúde, dentre os (as) idosos (as) que referiram perceber sua condição de saúde com regular ou ruim 67,2\% eram mulheres. Essa associação apresentou RR 1,26 (IC95\% 1,01;1,59, p= 0,04). A dimensão autopercepção de saúde também apresentou correlação significativa com todas as demais dimensões avaliadas pelo instrumento. Autopercepção negativa de saúde, na população de estudo, pode ser considerada um preditor de fragilidade pelo modelo de regressão logística (RR 2,17 IC 95\% 1,81;2,48, p= 0,0001). 
Por outro lado, avaliando os (as) idosos (as) robustos da amostra, percebe-se que maioria eram mulheres $(55,9 \%)$, com faixa etária entre 60 e 79 anos $(88,7 \%)$, de raça não-branca $(69,2 \%)$, de baixa escolaridade $(55,4 \%)$, vivendo sozinhos ou só com cônjuge (51,5\%). Estatisticamente, todas as características sociodemográficas analisadas apresentaram associação significativa com robustez, exceto a raça. Vale ressaltar que o sexo feminino apresentou associação significativa tanto com robustez quanto com fragilidade.

Tabela 4 - Associação entre sexo e os domínios do IVCF-20 em idosos (as) da UBS, Bahia, Brasil, 2020 (n=354).

\begin{tabular}{|c|c|c|c|c|}
\hline & $\begin{array}{l}\text { Fem. } \\
\text { n }(\%)\end{array}$ & $\begin{array}{l}\text { Masc. } \\
\text { n (\%) }\end{array}$ & $\begin{array}{l}\text { Total } \\
\text { n }(\%)\end{array}$ & P-valor \\
\hline Autopercepção da saúde & & & & $<0,0001$ \\
\hline Excelente/Muito Boa/Boa & $100(45,7 \%)$ & $77(57 \%)$ & $177(50 \%)$ & \\
\hline Regular/ruim & $119(54,3 \%)$ & $58(43 \%)$ & $177(50 \%)$ & \\
\hline AIVD & & & & $<0,00001$ \\
\hline Independente & $168(76,7 \%)$ & $100(74,1 \%)$ & $268(75,7 \%)$ & \\
\hline Com dependência & $51(23,3 \%)$ & $35(25,9 \%)$ & $86(24,3 \%)$ & \\
\hline ABVD & & & & 0,515 \\
\hline Independente & $208(95 \%)$ & $126(93,3 \%)$ & $334(94,3 \%)$ & \\
\hline Com Dependência & $11(5 \%)$ & $9(6,7 \%)$ & $20(5,7 \%)$ & \\
\hline Cognição & & & & 0,137 \\
\hline Sem déficit & $127(58 \%)$ & $89(65,9 \%)$ & $216(61 \%)$ & \\
\hline Com déficit & $92(42 \%)$ & $46(34,1 \%)$ & $138(39 \%)$ & \\
\hline Humor & & & & 0,215 \\
\hline Normal & $135(61,6 \%)$ & $92(68,1 \%)$ & $227(64,1 \%)$ & \\
\hline Alterado & $84(38,4 \%)$ & $43(31,9 \%)$ & $127(35,9 \%)$ & \\
\hline Alcance, Preensão e Pinça & & & & 0,141 \\
\hline Capaz & $194(88,6 \%)$ & $126(93,3 \%)$ & $320(90,4 \%)$ & \\
\hline Incapaz & $25(11,4 \%)$ & $9(6,7 \%)$ & $34(9,6 \%)$ & \\
\hline Capacidade Aeróbica e Muscular & & & & 0,913 \\
\hline Normal & $135(61,6 \%)$ & $84(62,2 \%)$ & $219(61,9 \%)$ & \\
\hline Alterado & $84(38,4 \%)$ & $51(37,8 \%)$ & $135(38,1 \%)$ & \\
\hline Marcha & & & & 0,810 \\
\hline Normal & $140(63,9 \%)$ & $88(65,2 \%)$ & $228(64,4 \%)$ & \\
\hline Alterada & $79(36,1 \%)$ & $47(34,8 \%)$ & $126(35,6 \%)$ & \\
\hline Continência & & & & 0,668 \\
\hline Normal & $171(78,1 \%)$ & $108(80 \%)$ & $279(78,8 \%)$ & \\
\hline Alterada & $48(21,9 \%)$ & $27(20 \%)$ & $75(21,2 \%)$ & \\
\hline Visão & & & & 0,172 \\
\hline Sem problema incapacitante & $193(88,1 \%)$ & $112(83 \%)$ & $305(86,2 \%)$ & \\
\hline Com problema incapacitante & $26(11,9 \%)$ & $23(17 \%)$ & $49(13,8 \%)$ & \\
\hline Audição & & & & 0,274 \\
\hline Sem problema incapacitante & $198(90,4 \%)$ & $117(86,7 \%)$ & $315(89 \%)$ & \\
\hline Com problema incapacitante & $21(9,6 \%)$ & $18(13,3 \%)$ & $39(11 \%)$ & \\
\hline Comorbidades Múltiplas & & & & 0,024 \\
\hline Não & $144(65,7 \%)$ & $104(77 \%)$ & $248(70,1 \%)$ & \\
\hline Sim & $75(34,3 \%)$ & $31(23 \%)$ & $106(29,9 \%)$ & \\
\hline Classificação Final & & & & 0,001 \\
\hline Robustos(as) & $103(47 \%)$ & $81(60 \%)$ & $184(52 \%)$ & \\
\hline Pré-frágeis & $86(39,3 \%)$ & $28(20,7 \%)$ & $114(32,2 \%)$ & \\
\hline Frágeis & $30(13,7 \%)$ & $26(19,3 \%)$ & $56(15,8 \%)$ & \\
\hline
\end{tabular}

Fonte: Elaborado pelos autores (2021). 
Tabela 5 - Medidas de associação brutas e ajustadas entre fragilidade e a idade, o sexo, a raça/cor e a presença de polifarmácia, Vitória da Conquista, Bahia, Brasil, 2020 (n=354).

\begin{tabular}{lcccc}
\hline & $\begin{array}{l}\text { Razão de Prevalência } \\
\text { Bruta (IC 95\%) }\end{array}$ & P-valor & $\begin{array}{l}\text { Razão de Prevalência } \\
\text { Ajustada (IC 95\%)* }\end{array}$ & P-valor \\
\hline Idade & 1,80 & $<0,001$ & 1,85 & $<0,001$ \\
& $(1,49-2,03)$ & & $(1,52-2,10)$ & \\
Sexo & 1,34 & 0,01 & 1,29 & 0,08 \\
& $(1,06-1,60)$ & & $(0,96-1,61)$ & \\
\hline Raça/cor & 0,78 & 0,27 & 0,94 & 0,67 \\
& $0,67-1,09)$ & & $(0,69-1,20)$ & \\
\hline Polifarmácia & 2,58 & $<0,001$ & 2,59 & $<0,001$ \\
& $(2,30-2,75)$ & & $(2,29-2,77)$ & \\
\hline
\end{tabular}

*Ajustado por Idade, Sexo, Raça/cor e Polifarmácia. Fonte: Elaborado pelos autores (2021).

\section{Discussão}

Nosso estudo revelou uma prevalência de fragilidade na população estudada de 15,8\%, sendo que 47,5\% da amostra apresenta algum grau de fragilidade (frágeis + pré-frágeis). Os dados são compatíveis com outros estudos mais recentes realizados com idosos brasileiros na comunidade, nos quais a prevalência de fragilidade variou de $11 \%$ e 28,2\% (Araujo Jr. et al., 2019; Bolina et al., 2019; Brigola et al., 2020; Carneiro et al., 2020; Freitas et al, 2020; Gross et al., 2018; Jesus et al., 2017; Lenardt et al., 2016; Lins et al., 2019; Maia et al., 2020; Oliveira et al., 2020; Sousa et al., 2018) e a prevalência de algum grau de fragilidade variou de 48,1\%, a 59,1\% (Alexandrino et al., 2019 \& Oliveira et al., 2020). Quase metade da amostra em alguma condição de fragilidade evidencia uma prioridade na rede de saúde que demanda cuidados e suporte bem como indica a necessidade de assistência tanto pela equipe de saúde quanto em domicílio para prevenir o desenvolvimento e o agravamento dessa vulnerabilidade clínica-funcional.

A prevalência de fragilidade aumenta conforme a idade e se mostrou maior entre os idosos (as) com 80 anos ou mais e esse grupo de longevos frágeis apresentou maior dependência para atividades de vida diária tanto básicas quanto instrumentais, pior mobilidade e comunicação e maior presença de comorbidades múltiplas. Essa associação é consistente e amplamente observada na literatura, em certa medida, esperada, uma vez que o envelhecimento se dá através da redução da reserva fisiológica mediante danos moleculares e celulares, variáveis conforme as características ambientais e sociais (Carneiro et al., 2020; Gross et al., 2018; Lins et al., 2019; Perracini \& Fló, 2019).

Ser longevo no Brasil é um desafio, em especial os que apresentam déficits na capacidade funcional, mobilidade, comunicação e comorbidades. Esse é o subgrupo etário que mais cresce, é predominantemente SUS dependente e deve ser preferencial nos modelos de decisão adotados pela equipe de ESF. Para ampliar a decisão diagnóstica, reconhecer as dimensões de saúde que mais comumente se associam à fragilidade pode resultar em reconhecimento das principais condições que acometem a população e na elaboração de estratégias de prevenção e reabilitação efetivas (Mendonça et al., 2020).

Sexo feminino e fragilidade também se associaram, corroborando o que se encontra na literatura contemporânea sobre a temática. Mulheres tendem a viver mais, superando as mortes precoces em consequência de acidentes e violência, além de, durante a idade reprodutiva, terem maior acesso à rede de assistência à saúde. Em revisão sistemática sobre fragilidade, utilizando-se o Frailty Index Escore, ficou demonstrado que as mulheres apresentam escores mais elevados em todas as faixas etárias, porém, elas toleram os eventos adversos relacionados à fragilidade melhor e, portanto, o tempo médio de sobrevida com incapacidade é maior do que o dos homens (Fernandes et al., 2013; Gordon et al., 2017; Lins et al., 2019; Perracini \& Fló, 2019).

A feminização da velhice é um fenômeno observado no envelhecimento populacional e se discute que para mulheres viver mais não é sinônimo de viver bem. É importante salientar que a combinação entre condições de dependência e pobreza torna as mulheres mais vulneráveis à negligência, ao abandono, a abusos e a maus tratos pois sabe-se que em relação aos homens 
idosos, elas têm menor probabilidade de receber cuidado. Segundo dados da Pesquisa Nacional de Saúde do IBGE e da pesquisa Estudo Longitudinal de Saúde dos Idosos Brasileiros-Elsi (Camarano, 2020), na população idosa a maioria das necessidades de cuidados é das mulheres e a demanda está concentrada nos estratos menos escolarizados e mais pobres. Embora as idosas demandem maior cuidado e apresentem as maiores dificuldades para o desempenho de tarefas de vida diária, a maior proporção de insuficiência de cuidado está entre as mulheres, mais jovens e em arranjos domiciliares maiores (Giacomin et al, 2018; Camarano, 2020).

Os fatores socioculturais, então, também estão associados à fragilidade em idosos(as), e aqui enfatizamos a baixa escolaridade, visto ser o estado da Bahia aquele com maior número de pessoas analfabetas do país. A escolaridade é um fator de risco modificável para fragilidade e tem impacto também em outras dimensões de saúde, como cognição e humor. Estudos também apontam que pessoas com baixa escolaridade têm menores chances de reabilitação e maiores de fragilização no curto prazo. Além disso, a baixa escolaridade está frequentemente associada a hábitos de vida considerados não saudáveis (Etman et al., 2015; Ikeda et al., 2019; Lee et al., 2018). As diferenças educacionais e ocupacionais continuam a ter impacto na fragilidade com o envelhecimento, efeito reconhecido como sendo maior que o impacto da renda (Hoogendijk et al., 2014; Stolz et al., 2017).

Com relação às condições de moradia, pessoas idosas que vivem sozinhas ou somente com cônjuges apresentaram menores índices de fragilidade. A literatura atual apresenta resultados conflitantes nesse aspecto. Uma revisão sistemática e meta-análise publicada em 2020 demonstrou que pessoas idosas vivendo sozinhas são mais propensos a apresentar fragilidade, especialmente os idosos do sexo masculino (Kojima et al., 2020). Contudo, morar sozinho ou só com cônjuge também demanda manutenção da funcionalidade e renda suficiente. Em nosso estudo observamos que as pessoas idosas que residem com outros familiares apresentam maior prevalência de fragilidade e maior grau de dependência funcional, o que indica que as necessidades de cuidado têm potencial de reorganização das configurações familiares (Rabelo et al., 2020).

A fragilidade também estava associada com polifarmácia, com polipatologia, com histórico de internação recente e com autopercepção negativa de saúde. Estudos indicam maior probabilidade de desenvolver fragilidade em pacientes idosos em que se identificou polifarmácia (Gutiérrez-Valencia, et al., 2018) e que quanto maior o número de medicamentos (5 ou mais), maior o risco (Maia et al., 2020; Pagno et al., 2018). Desta forma, revisões rotineiras das prescrições visando reduzir o número de medicações utilizadas constitui estratégia importante para a redução da fragilidade e melhora da qualidade de vida de pessoas idosas da comunidade.

A polipatologia já se apresenta em uma série de instrumentos que trazem o número de patologias como um dos critérios para definição de fragilidade. Vale ressaltar que a prevalência de doenças crônicas aumenta com a idade e um número significativo de doenças prevalentes cursam com perda de capacidade aeróbica e muscular, além de maior necessidade de assistência médica e uso de medicações. Idosos com polipatologia também estão mais suscetíveis a hospitalizações e frequentemente evoluem com declínio funcional (Ferrucci \& Fabri, 2018; Zazzara et al., 2019).

A autopercepção de saúde se associa ao desempenho funcional, cognitivo e emocional e idosos(as) com fragilidade frequentemente avaliam negativamente sua saúde (Carneiro, 2020a; Loch et al., 2015; Tovel et al., 2019). A saúde percebida tem sido descrita como um parâmetro confiável de capacidade funcional e mortalidade e sua classificação como negativa reflete a avaliação que a pessoa idosa faz de seus aspectos físicos, cognitivos e emocionais (Mendonça et al., 2020).O reconhecimento de idosos(as) com autopercepção negativa de saúde no âmbito da ESF pode ser considerado um importante impulsionador de estratégias comunitárias e criação de grupos operativos para promoção da saúde e convívio social na população. Nesse estudo, as mulheres fizeram uma pior avaliação da sua própria saúde.

Quanto à raça, em nossa amostra, não houve associação estatística para fragilidade. Os efeitos da cor/raça na saúde da população idosa brasileira ainda apresentam divergências entre os estudos (Oliveira, Thomaz \& Silva, 2014, Silva, 2019; Silva 
et al., 2018). Pode refletir as limitações da autoclassificação racial em nosso país e o efeito de seletividade decorrente da ocorrência de viés de prevalência e de sobrevivência, uma vez que idosos de pior status socioeconômico apresentam menor sobrevida (Oliveira et al., 2014). A ausência de associação entre raça/cor da pele e fragilidade nesse estudo não significa que não existam desigualdades raciais na saúde e nas condições gerais de vida de pessoas idosas. Estas devem ser devidamente reconhecidas nos sistemas de saúde baseados em princípios de equidade

\section{Conclusão}

Os resultados indicaram que quase metade das pessoas idosas apresentaram algum grau de fragilidade e o grupo com maior risco foram os longevos, as mulheres, os com baixa escolaridade, os que coabitavam com outros familiares ou só com cônjuge, com polifarmácia, com polipatologia, com histórico de internação recente, e com autopercepção negativa de saúde. Reconhecer e encaminhar adequadamente pessoas idosas em situação de vulnerabilidade clínico-funcional, bem como estabelecer estratégias eficientes, considerando os fatores de risco existentes na população assistida pela atenção básica, são importantes aliados na prevenção do desenvolvimento e do agravamento da fragilidade em pessoas idosas.

Este estudo contribui para ampliar os conhecimentos sobre a fragilidade em contextos diferentes, como as pessoas idosas do território baiano, e que inclua a variável raça/cor da pele. Por tratar-se de estudo com delineamento transversal não é possível, com nossos resultados, estabelecer relações de causalidade, o que constitui uma limitação. No entanto, traz importantes considerações sobre as características epidemiológicas da população estudada. Estudos longitudinais podem ser estimulados e mais evidências surgirem. Além disso, o estudo traz apontamentos para a elaboração de políticas de saúde pública voltadas para uma população que, até então, esteve pouco em evidência no âmbito municipal. Nesse sentido, espera-se poder estimular outras equipes de UBS locais a realizar o rastreamento em seus idosos, ampliando assim o impacto positivo na qualidade de assistência à saúde da pessoa idosa no município e favorecendo as políticas públicas voltadas para essa população.

Cabe destacar que, a presente pesquisa ocorreu em período imediatamente anterior ao início da pandemia do Coronavírus, o que trouxe à tona uma infinidade de questões de saúde pública, especialmente na população idosa. O reconhecimento desse grupo populacional como prioritário para o sistema de saúde tornou-se imperativo. Pesquisas como a nossa auxiliam as gestões municipais a identificar as dimensões de saúde envolvidas na qualidade de vida dos idosos, além de estimular as equipes a conhecerem suas populações adstritas para desenvolver ações de promoção e prevenção no âmbito local. Equipes que anteriormente mantinham seus cadastros de idosos atualizados se beneficiaram nesse período de isolamento social imposto pela pandemia e conseguiram minimizar seus impactos.

A presente pesquisa tem o potencial de alavancar trabalhos futuros visando a criação e/ou aprimoramento de ferramentas de rastreio da fragilidade, ou até mesmo a introdução de instrumentos já respaldados como o IVCF-20 de maneira ampla no SUS, com o objetivo de rastrear os idosos para fomentar a promoção e prevenção em saúde, adiar/frear o avanço dessa síndrome e/ou fazer os devidos encaminhamentos para o(s) especialista (s), como é o caso do geriatra, com as devidas adaptações sociais. Outra possibilidade são novos estudos comparativos e/ou a longo prazo, que possibilitem essa análise causal, que acarretará uma economia de custos para os cofres públicos, uma maior autonomia desse grupo, através do estabelecimento de ações e políticas de saúde efetivas para a comunidade, com a contribuição das instituições acadêmicas.

\section{Referências}

Alexandrino, A, Cruz, E., Medeiros, P., Oliveira, C., Araújo, D. \& Nogueira, M. (2019). Evaluation of the clinical-functional vulnerability index in older adults. Rev. Bras. Geriatr. Gerontol., 22(6), 01-12.

Araujo Jr,, F. B., Machado,I., Santos-Orlandi, A., Pergola-Marconato, A., Pavarini, S.\& Zazzetta, M. (2019). Fragilidade, perfil e cognição de idosos residentes em área de alta vulnerabilidade social. Cien Saúde Colet, 24(8), 3047-3056. 
Bolina, A. F., Rodrigues, R., Tavares, D. \& Haas, V. (2019). Factors associated with the social, individual and programmatic vulnerability of older adults living at home. Rev. Esc. Enferm. USP, 53(1), 01-09.

Brasil. (2019). Ministério da Saúde. Nota técnica para organização da rede de atenção à saúde com foco na atenção primária à saúde e na atenção ambulatorial especializada - Saúde da pessoa idosa. Hospital Israelita Albert Einstein. Ministério da Saúde.

Brasil. (2021). Ministério da Saúde. Portaria MS/GM n 2.528 de 19 de outubro de 2006. Aprova a Política Nacional de Saúde da Pessoa Idosa. Brasília: MS, 2006. https://bvsms.saude.gov.br/bvs/saudelegis/gm/2006/prt2528_19_10_2006.html.

Brigola, A. G., Ottaviani, A., Carvalho, D., Oliveira, N., Souza, E. \& Pavarini, S. (2020). Association between cognitive impairment and criteria for frailty syndrome among older adults. Arq. Neuro-Psiquiatr., 78(1), 2-8.

Carneiro, J. A. Gomes, C., Durães, W., Jesus, D., Chaves, K., Lima,C., Costa,F. \& Caldeira, A. (2020). Autopercepção negativa da saúde: prevalência e fatores associados entre idosos assistidos em centro de referência. Cien Saúde Colet, 25(3), 909-918.

Carneiro, J. A., Souza, A.S., Maia, L.C., Costa, F.M., Moraes, E.N. \& Caldeira, A.P. (2020). Frailty in community-dwelling older people: comparing screening instruments. Rev. Saúde Pública, 54(119), 15-31.

Castro-Costa, E., Lima-Costa, M. F., Andrade, F. B. de, Souza Junior, P. R. B. de \& Ferri, C. P. (2018). Cognitive function among older adults: ELSI-Brazil results. Rev. Saúde Pública, 52(2), 4s.

Etman, A., Kamphuis, C., Cammen, T., Burdorf, A. \& Lenthe, F. (2015). Do lifestyle, health and social participation mediate educational inequalities in frailty worsening? Eur. J. Public Health., England, 25(2), 345-50.

Faller, J. W., Pereira, D., Souza, S., Nampo, F., Orlandi, F. \& Matumoto, S. (2019). Instruments for the detection of frailty syndrome in older adults: A systematic review. PLoS ONE, Maryland, 14(4), 01-10.

Fernandes, H. C. L., Gaspar, J., Yamashita, C., Amendola, F., Alvarenga, M. \& Oliveira, M. (2013). Avaliação da fragilidade de idosos atendidos em uma unidade da Estratégia Saúde da Família. Texto Contexto - Enferm., 22(2), 423-431.

Ferrucci, L. \& Fabbri E. (2018). Inflammageing: chronic inflammation in ageing, cardiovascular disease, and frailty. Nat. Rev. Cardiol., 15(9), 505-522.

FIOCRUZ. (2020). Sistema de Indicadores de saúde e Acompanhamento de Políticas Públicas do Idoso (SISAP-Idoso). https://sisapidoso.icict.fiocruz.br.

Fontelles, M. J., Simões, M. G., Farias, S. H., \& Fontelles, R. G. S. (2009). Metodologia da pesquisa científica: diretrizes para a elaboração de um protocolo de pesquisa / Metodologia da pesquisa científica: diretrizes para elaboração de um protocolo de pesquisa. Rev. par. med. p.23.

Freitas, F, F. Q., Rocha, A., Moura, A. \& Soares, S. (2020). Fragilidade em idosos na Atenção Primária à Saúde: uma abordagem a partir do geoprocessamento. Cien Saúde Colet, 25(11), 4439-4450.

Gil, A. C. (2002). Como elaborar projetos de pesquisa. (4a ed.), Atlas.

Gordon, E. H, Peel, N.M., Samanta, M., Theou, O., Howlett, S. \& Hubbard, R. (2017). Sex differences in frailty: A systematic review and meta-analysis. Exp. Gerontol., 89(1), 30-40.

Gross, C. B., Kolankiewicz, A., Schmidt, C. \& Berlezi, E. (2018). Níveis de fragilidade de idosos e sua associação com as características sociodemográficas. Acta Paul. Enferm., 31(2), 209-216.

Gutiérrez-Valencia, M. Izquierdo, M., Cesari, M, Casas-Herrero, Á, Inzitari, M \& Martínez-Velilla, N. (2018). The relationship between frailty and polypharmacy in older people: A systematic review. Br. J. Clin. Pharmacol., 84(7), 1432-1444.

Hoogendijk, E. O., Hout,H., Heymans,M., Horst,H., Frijters, D., Groenou, D., Deeg, D. \& Huisman, M. (2014). Explaining the association between educational level and frailty in older adults: results from a 13-year longitudinal study in the Netherlands. Ann. Epidemiol., 24(7), 538-44.

Ikeda, T., Tsuboya, T., Jun Aida, J., Matsuyama, Y., Koyama, S., Kemmyo Sugiyama,K., Kondo, K \& Osaka, K. (2019). Income and education are associated with transitions in health status among community-dwelling older people in Japan: the JAGES cohort study. Fam. Pract., 18(36), 713-722.

Instituto Brasileiro de Geografia e Estatística (IBGE). (2019). IBGE Cidades. https://cidades.ibge.gov.br/brasil/ba/vitoria-da-conquista/panorama.

Jesus, I. T. M., Orlandi, A., Grazziano, E. \& Zazzetta, M. (2017). Fragilidade de idosos em vulnerabilidade social. Acta Paul. Enferm., 30(6), 614-620.

Khezrian, M., Myint, P., McNei, C. \& Murray, A. (2017). A Review of Frailty Syndrome and Its Physical, Cognitive and Emotional Domains in the Elderly. Geriatrics, 2(4), 36-46.

Kojima, G. (2019). Increased costs associated with frailty among community-dwelling older people: A systematic review and meta-analysis. Arch. Gerontol. Geriatr., 84(1), 01-10.

Kojima, G., Iliffe, S., Jivraj, S. \& Walters, K. (2016). Association between frailty and quality of life among community-dwelling older people: A systematic review and meta-analysis. J. Epidemiol. Community Health, 70(7), 716-21.

Kojima, G., Liljas,A. \& Iliffe,S. (2019). Frailty syndrome: implications and challenges for health care policy. Risk Manag. Healthc. Policy, 12(1), 23-30.

Kojima, G., Taniguchi, Y., Kitamura, A., \& Fujiwara, Y. (2020). "Is living alone a risk factor of frailty? A systematic review and meta-analysis.” Ageing research reviews, 59.

Lakatos, E. M., \& Marconi, M. A. (2003). Fundamentos de metodologia científica. (5a ed.), Atlas. 
Lee, D. R., Santo, E., Lo, J., Weintraub, M., Patton, M. \& Gordon, N. (2018).Understanding functional and social risk characteristics of frail older adults: a cross-sectional survey study. BMC Fam. Pract., 19(1), 170-81.

Lenardt, M. H., Carneiro, N., Binotto, M., Willig, M., Lourenço, T. \& Albino, J. (2016). Fragilidade e qualidade de vida de idosos usuários da atenção básica de saúde. Rev. Bras. Enferm., 69(3), 478-483.

Lins, M. E. M., Marques, A., Leal, M. \& Barros, R. (2019). Risco de fragilidade em idosos comunitários assistidos na atenção básica de saúde e fatores associados. Saúde Debate, 43(121), 520-529.

Loch, M. R. Souza, R., Mesas, A., González, A. \& Rodriguez-Artalejo, F. (2015). Associação entre capital social e autopercepção de saúde em adultos brasileiros. Rev. Saúde Pública, 49(53), 01-09.

Maia, L. C., Colares, T., Moraes, E., Costa, S. \& Caldeira, A. (2020). Robust older adults in primary care: factors associated with successful aging. Rev. Saúde Pública, 54(35), 01-15.

Maia, L. C., Costa, S.M \& Caldeira, A.P. (2020). Fragilidade em idosos assistidos por equipes da atenção primária. Cien Saúde Colet, 25(12), 5041-5050.

Miyamura, K., Fhon, J., Wilmer, A., Fuentes-Neira, W., Silveira, R. C. \& Rodrigues, R. (2019). Frailty syndrome and cognitive impairment in older adults: systematic review of the literature. Rev. Latino-Am. Enfermagem, 27(1), 01-12.

Moraes, E. N. (2012). Atenção à saúde do idosos: Aspectos Conceituais. Brasília: Organização Pan Americana da Saúde.

Nascimento, P. P. \& Batistoni, S. S. T. (2019). Depressão e fragilidade na velhice: uma revisão narrativa das publicações de 2008-2018. Interface (Botucatu), 23(1), 01-16.

Oliveira, C. E. S., Felipe, S., Silva, C., Carvalho, D., Silva-Júnior, F., Figueiredo, M., Santos, A. \& Gouveia, M. (2020). Vulnerabilidade clínico-funcional de idosos em um centro de convivência. Acta Paul. Enferm., 33(1), 01-08.

Organização Mundial da Saúde (OMS). (2015). Relatório mundial de envelhecimento e saúde. Genebra.

Pagno, A. R., Gross, C. B., Gewehr, D. M., Colet, C. F., \& Berlezi, E. M. (2018). Drug therapy, potential interactions and iatrogenesis as factors related to frailty in the elderly. Rev. Bras. Geriatr. Gerontol. 21(5), 588-596.

Perracini M. R., \& Fló C. M. (2019). Funcionalidade e envelhecimento. (2a ed.), Guanabara Koogan.

Placideli, N. \& Nemes, M. (2020). Evaluation of comprehensive care for older adults in primary care services. Rev. Saúde Pública, 54(1), 01-14.

Romero, D. E. \& Silva, D.R. (2021) Idosos no contexto da pandemia da COVID-19 no Brasil: efeitos nas condições de saúde, renda e trabalho. Cadernos de Saúde Pública. 37(3). https://doi.org/10.1590/0102-311X00216620.

Sousa, J. A. V., Lenardt, M., Grden, C., Kusomota, L., Dellaroza, M. \& Betiolli, S. (2018). Physical frailty prediction model for the oldest old. Rev. Latino-Am. Enfermagem, 26(1), 01-13.

Stolz, E, Mayer,H., Waxenegger, A., Rásky, É. \& Freidl,W. (2017). Impact of socioeconomic position on frailty trajectories in 10 European countries: evidence from the Survey of Health, Ageing and Retirement in Europe (2004-2013). J. Epidemiol. Community Health, 71(1), 73-80.

Tovel, H. Carmel, S. \& Raveis, V. (2019). Relationships Among Self-perception of Aging, Physical Functioning, and Self-efficacy in Late Life. J. gerontology, 74(1), 212-221.

Zazzara, M. B., Vetrano, D., Carfi, A. \& Onder, G. (2019).Frailty and chronic disease. Panminerva Med. 61(4), 486-492. 